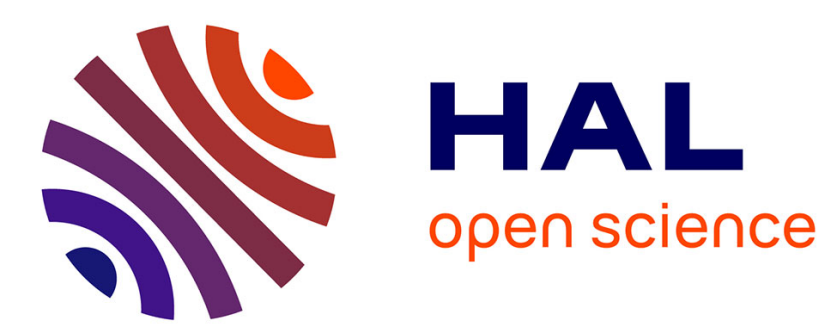

\title{
De l'alcoolisme à l'abstinence: le choc biographique comme rupture symbolique
}

\author{
Omar Zanna, Hugues Pentecouteau
}

\section{To cite this version:}

Omar Zanna, Hugues Pentecouteau. De l'alcoolisme à l'abstinence: le choc biographique comme rupture symbolique. Nouvelle revue de psychosociologie, 2013, 1 (15), pp.289-304. 10.3917/nrp.015.0289 . hal-01105004

\section{HAL Id: hal-01105004 https://hal.science/hal-01105004}

Submitted on 4 Jun 2019

HAL is a multi-disciplinary open access archive for the deposit and dissemination of scientific research documents, whether they are published or not. The documents may come from teaching and research institutions in France or abroad, or from public or private research centers.
L'archive ouverte pluridisciplinaire HAL, est destinée au dépôt et à la diffusion de documents scientifiques de niveau recherche, publiés ou non, émanant des établissements d'enseignement et de recherche français ou étrangers, des laboratoires publics ou privés. 


\section{DE L'ALCOOLISME À L'ABSTINENCE : LE CHOC BIOGRAPHIQUE COMME RUPTURE SYMBOLIQUE}

Omar Zanna et Hugues Pentecouteau

ERES Nouvelle revue de psychosociologie

Pour citer cet article : Zanna Omar et Pentecouteau Hugues, « De l'alcoolisme à l'abstinence : le choc biographique comme rupture symbolique », Nouvelle revue de psychosociologie, 2013/1 n 15, p. 289-304. DOI :

10.3917/nrp.015.0289 



\title{
Études
}

\section{De l'alcoolisme à l'abstinence : le choc biographique comme rupture symbolique}

\author{
Omar Zanna \\ Hugues Pentecouteau
}

Entre 2007 et 2009, nous avons réalisé une centaine d'observations au sein des groupes de parole animés par les Alcooliques anonymes (AA ${ }^{1}$. Parmi les AA rencontrés, beaucoup indiquent un moment ou un événement pour expliquer leur changement radical de rapport à la consommation d'alcool. Pourtant, l'analyse des entretiens recueillis a rapidement révélé que ce changement radical était en réalité à considérer comme le fruit d'un processus long et complexe qui ne peut se réduire à un événement spontané et individuel se produisant ex nihilo, comme beaucoup d'AA semblent le penser. Pour ces personnes qui ont - après la rencontre avec les AA rapidement arrêté de boire, l'abstinence s'apparente à une forme d'hapax existentiel - une forme d'insight -, c'est-à-dire à la découverte soudaine de la solution à un problème, un événement unique, une forme de révélation intervenant parfois sur le mode hystérique (pleurs, hallucinations, syncope) et déterminant, avec soudaineté, un cheminement radicalement différent. L'hapax existentiel est à considérer comme un événement, un « life-event » (Legrand, 1993) qui coupe en deux, dans les représentations des étapes d'une biographie, la réalité vécue d'un itinéraire (Jankélévitch, 1957). Toutefois, comme nous allons le montrer, l'hapax existentiel auquel certains AA font référence comme à une rupture symbolique pour expliquer l'entrée en abstinence nécessite un cheminement en amont et en aval de la rencontre avec les AA. En d'autres termes, si le savoir indigène est nécessaire aux individus pour s'orienter dans le monde dans 
Omar Zanna, sociologue, maître de conférences (HDR), Groupe de recherche : violences, identités, politiques \& sport (VIP\&S), université du Maine (Le Mans).

Hugues Pentecouteau, sociologue, Centre de recherche sur l'éducation, les apprentissages et la didactique (CREAD), maître de conférences, université de Rennes 2.

1. Les AA, c'est 100000 groupes locaux dans 150 pays, soit environ 2 millions d'individus, selon les évaluations faites par les AA. La France compte 630 groupes dont 53 localisés en Bretagne.

lequel ils vivent et pour l'interpréter, le savoir savant du chercheur, cet « observateur désintéressé » (Schütz, 1987), ne peut s'en contenter pour rendre compte des motifs d'agir des individus. Là où les $A A$, en évoquant un changement radical, tombent dans le piège de ce que Norbert Elias (1991) nomme la Zustandreduktion, qui consiste à réduire des processus à des états, le sociologue, lui, reconstruit le processus de l'arrêt de boire, dont l'événement retenu par les AA pour l'expliquer n'est, en réalité, qu'un facteur parmi d'autres.

Pour rendre compte de ce processus d'arrêt de boire, comparable à une conversion, nous interrogerons ici le « cas » Camille. Camille est un alcoolique anonyme âgé de 80 ans, abstinent quasiment du jour au lendemain (dans ses représentations), depuis une trentaine d'années. Nous tenterons de comprendre ses comportements, ses opinions, ses représentations qui s'appuient sur des valeurs et des croyances, afin de les analyser et de les mettre à l'épreuve de la thèse de l'hapax existentiel pour en tirer une signification dépassant le niveau individuel (Hoggart, 1991). Car si l'individu est une partie de la société, la société est présente dans chaque individu en tant que tout, notamment au travers de son langage, de sa culture, des normes... C'est ce dont les lignes qui suivent voudraient rendre compte, non sans avoir au préalable évoqué les conditions de recueil des données.

\section{DEUX SOCIOLOGUES ENTRENT CHEZ LES AA}

Pour nous immerger dans le « monde » social des AA, nous avons opté pour une observation mêlant les approches participative et ethnographique que l'on ramènera, selon l'expression de Mills (1983), à l'idée d'artisanat intellectuel. II nous a donc fallu trouver des groupes et négocier notre place pour y entrer. Le plus souvent, nous nous sommes présentés en début de séance, devant l'ensemble des participants, afin d'indiquer notre démarche. Le sociologue, bien qu'agent du désenchantement (Weber, 1963), est plutôt accueilli avec curiosité et intérêt.

Si dans les groupes des AA - comme dans toutes les organisations sociales - le principe maussien du don et du contre-don est opératoire, dans ce lieu le sens habituel de déplacement des biens symboliques, entre observateurs et observés, est inversé. À plusieurs reprises nous avons été remerciés pour notre présence et pour l'intérêt que nous leur portions. Cette inversion de la dynamique de réciprocité s'explique notamment par la lutte pour la reconnaissance (Honneth, 2002) des AA qui, pour certains, s'apparente à une véritable croisade contre l'alcool. Ce faisant, la simple présence d'une personne étrangère au groupe, qui plus est lorsqu'il s'agit d'un chercheur, est appréhendée comme un don qu'il faut honorer sans attendre. Parmi d'autres 
facteurs, cette volonté de rétablir l'identité blessée, autrement dit cette lutte permanente pour la reconnaissance, explique cette inversion du sens de la réciprocité de l'échange. Tout se passe comme si la présence du chercheur s'apparentait à un don apprécié qui rend, point important, le contre-don de la parole de l'enquêté plus aisé et sans doute moins fardé. Les AA saisissent la présence du chercheur pour témoigner et se faire entendre, pour porter leurs expériences au-delà de leur cercle.

$\mathrm{Au}$ cours de cette recherche, nous avons effectué une centaine d'observations (de

1 heure 30 à 5 heures) à Brest et à Rennes. Les deux tiers d'entre elles ont été volontairement réalisés au sein du même groupe. Ces groupes auxquels nous nous sommes " attachés »- nous reprenons ici la notion, issue du vocabulaire des AA, de « groupe d'attache ${ }^{1}$ »- se sont révélés par la suite être des éléments essentiels pour la recherche. Le fait de retrouver les mêmes personnes d'une réunion à l'autre facilite l'enquête dans la mesure où le travail qui consiste à estomper la méfiance propre à toutes les nouvelles situations de face-à-face n'est pas à refaire. La banalisation des rencontres procure un confort psychologique ; il permet de dépasser les échanges convenus des premières entrevues. La régularité a fait du chercheur un « ami naturel » qui peut diffuser la « bonne » parole des AA. II devient, d'une certaine manière, « l'un des leurs » (Kessel, 1960) et continue à l'être dans la mesure où il participe à des réunions. Précisons enfin que des entretiens formels et informels ont été effectués, mais uniquement à titre complémentaire.

Ce matériau permet d'entrer dans une démarche interprétative « en exprimant le réel » (Durkheim, 1977) et en cherchant à porter un autre regard sur les AA. Nous avons appréhendé ces groupes en postulant que nous avions en face de nous des êtres sociaux qui construisent leur identité autour de la consommation d'alcool et d'une démarche d'abstinence. Précisons que nous ne les regardons pas comme des malades, des déviants ou des personnes en dehors de la norme, mais comme des individus qui ont utilisé l'alcool comme un mode singulier de régulation de leurs devenirs individuels et qui sont en passe de trouver un nouveau mode de régulation avec le groupe de parole (Le Grand, 1997).

\section{UNE SOCIOLOGIE DE L'ABSTINENCE}

En novembre 2007, lorsque nous avons entrepris de travailler sur l'abstinence, nous avons élaboré une problématique, un cadre général de réflexion. II portait sur l'identification et la compréhension de dispositions permettant à un individu, alcoolique, d'entrer et de demeurer dans un parcours d'abstinence. Ce parti pris théorique fut notre point de départ. Pour autant, nous savions qu'il ne résisterait pas longtemps aux faits, car si nous avions besoin d'un modèle théorique pour définir un terrain d'investigation, nous ne doutions pas que nous serions amenés à envisager une autre problématique, théoriquement plus compréhensive. Cette façon d'envisager

\footnotetext{
1 . Un livret édité par AA Grapevine présente le groupe d'attache comme « le battement du cœur des AA », " la clé de l'unité » et le point de départ du rétablissement.
} 
la recherche sociologique est liée au fait que, l'un comme l'autre, nous avons toujours été fascinés par les travaux des chercheurs américains de l'école de Chicago et par leur méthodologie. Outsiders (Becker, 1985), Street Corner Society (Whyte, 1995), Becoming Deviant (Matza, 1964) - parmi d'autres nous présentaient une manière de faire de la recherche où l'on apprend du terrain tout en questionnant l'orientation du regard porté par le chercheur.

Dans cette logique appelant l'immersion, au sens plein du terme, dans le monde des alcooliques, le terrain a une vocation immense. II est une source de savoir et doit par conséquent être appréhendé dans une logique heuristique. C'est le principe même de la grounded theory qui célèbre la participation et l'investissement du chercheur en immersion, " à l'intérieur du monde social qu'il cherche à comprendre » : «Salissez vos pantalons », disait Robert Park à ses étudiants (Peneff, 1990, p. 51). Cette posture suppose par ailleurs la mise en œuvre d'un certain nombre de dispositions personnelles et d'empathies ${ }^{2}$ idiosyncrasiques, celles dont on a besoin dans toute relation sociale un peu imprévue, comme, par exemple, la capacité à entrer en relation avec des personnes présentant un ethos éloigné du nôtre, à gagner la confiance des enquêtés, à négocier une place sur le terrain, à y rester (Zanna, 2010). On ne peut en effet rien connaître de nos interlocuteurs si nous ne sommes pas a minima partie prenante, " actants » dans la recherche, autrement dit si nous ne nous sentons pas concernés par les situations étudiées (Barbier, 1997). Loin de l'idéal de neutralité qui fait fi de la subjectivité au profit des seules lois générales et impersonnelles censées régenter le social, la posture sociologique doit associer avec rigueur empathie et distance réflexive - l'objectivité sans perte d'affects.

La pratique de l'école de Chicago nous intéresse à un autre titre. Elle invite à apprendre du terrain sans forcément entrer dans une accumulation des données (Becker, 1985). On peut participer, ou pas, à des actions sociales ; travailler à partir de documents personnels (Thomas et Znaniecki, 1919). II est également possible de s'intéresser à des « cas », comme dans des approches ethnométhodologie (Garfinkel, 1967) ou psychiatriques (Foucault, 1973).

CAMILLE, UN « CAS » QUI MET AU JOUR LE PROCESSUS DU DEVENIR ABSTINENT

Souhaitant mettre à l'épreuve l'hypothèse de l'hapax existentiel pour comprendre le devenir abstème, le passage par la biographie, comme méthode d'investigation - parce qu'elle permet de faire apparaître les éléments qui rendent possible le phénomène étudié -, nous semblait indispensable. Nous avons donc travaillé sur un individu, en faisant du « cas

\footnotetext{
2. L'empathie est le fait de s'inscrire dans un processus qui consiste à percevoir le cadre de référence interne d'une personne avec précision (parfois plus clairement et plus explicitement qu'elle ne le ferait elle-même), de façon à le ressentir comme s'il était (analogue à) cette personne, sans toutefois y être identique (Hoffman, 2008). Être empathique, déployer ses antennes sociales pour apprécier l'autre, aller à sa rencontre, lui signifier qu'il peut parler parce que l'on est en état de l'écouter et de l'entendre, est sans conteste une des qualités majeures dans toute entreprise anthropologique.
} 
Camille » un dossier clinique (Garfinkel, 1967). Camille nous est vite apparu comme un « bon interlocuteur » (Bertaux, 1997) : il représente une ressource précieuse puisqu'il peut aider à percevoir, de l'intérieur et de manière très fine, ce qui s'y joue.

En optant pour le récit biographique, en nous appuyant sur une dizaine d'entretiens à intervalles réguliers avec la même personne (pendant seize mois), nous souhaitions partir d'une histoire personnelle afin d'y repérer un cheminement classique (au sens étymologique de « courant, habituel »). Se concentrer sur un cas extrême de changement d'être-au-monde permet de mettre en évidence les éléments du processus de devenir que l'on retrouve dans des cas moins extrêmes. L'analyse en profondeur d'un cheminement ou d'un phénomène peut en effet livrer exactement les mêmes aperçus que ceux obtenus par l'analyse de faits relativement superficiels illustrant l'étendue d'un grand nombre de cheminements ou de phénomènes.

\section{LA RENCONTRE DE CAMILLE AVEC LES AA : UN MOMENT DE SIDÉRATION}

L'analyse du discours révèle tout d'abord que l'hapax existentiel est à considérer comme un événement venant couper en deux la réalité vécue d'un itinéraire. En témoignent les propos de Camille :

« Ce jour-là (le 20 mars 1978), je savais qu'il y avait une réunion à Nanterre, et pour dire à quel point j'étais vraiment accro j'étais allé dans un bon restaurant et j'ai passé la soirée avec une bonne bouteille de bordeaux, je ne savais pas encore que c'était pour moi la dernière. C'était rare que je boive du bordeaux. J'étais bien chaud pour assister à ma première réunion, sans savoir vraiment ce qui m'attendait derrière la porte. J'y suis allé tout seul, comme un grand, et là, je crois que j'ai beaucoup pleuré. Je pleurais à entendre le discours des autres. J'étais très ému. Et c'était pour moi une grande découverte de voir ces gens qui, à tour de rôle, disaient qu'ils allaient bien et "qu'ils n'avaient pas bu aujourd'hui". Pour moi, qui avais bu ce jour-là, et qui allais très mal, cela a été un choc, dans le bon sens. Ce fut une grande bouffée d'espoir [...]. II m'a fallu huit jours à partir de cette première réunion pour arrêter définitivement l'alcool. »

Camille évoque ici un choc biographique dont il sortira radicalement transformé. La description faite de cette expérience radicale et fondatrice qui génère révélation et conversion n'est pas sans rappeler la transmutation vécue par saint Paul, saint Augustin, Pascal, Montaigne, La Mettrie, Rousseau et bien d'autres. Tous, comme Camille, évoquent pleurs, cris, syncope, étourdissement, palpitations... pour décrire le cyclone affectif dont leur corps est la proie et dont ils sortiront radicalement transformés. Les souffrances évoquées précipitent la mutation ontologique. Tel un rite initiatique, elles accompagnent « le passage d'un univers social à un autre, bouleversant d'un trait l'ancien rapport au monde [...]. Une expérience qui pourrait relever de la torture dans un autre contexte, possède ici une valeur de transformation positive et heureuse. Elle marque la mort symbolique et la renaissance » (Le Breton, 2010, p. 193). L'expérience, au sens étymologique d'épreuve, marque la mort symbolique et la renaissance. Subitement, pour Camille, ce lundi de mars 1978 et les jours qui suivent, avec sûreté et finesse indicible, quelque chose devient visible, audible, le sidère puis l'ébranle au 
plus intime de lui-même (Nietzsche, 1908). Cette expérience signe son entrée dans le groupe. Cela dit, quand bien même il évoque ce moment comme une révélation, cela ne signifie pas pour autant qu'il n'y ait pas été préparé. On ne part en effet jamais de rien, on invente toujours à partir de ce qui a eu lieu, de ce dont on a hérité. Le présent, et plus encore le futur, sont des purs produits du passé transfiguré.

UN PRÉSENT INSCRIT DANS LE PASSÉ

Dans le discours de Camille, le passé et, notamment, les relations avec un père autoritaire et exigeant sont régulièrement évoqués, comme pour lui permettre de rationaliser son histoire de vie, voire de justifier sa consommation excessive d'alcool. Passé, présent et futur sont donc étroitement intriqués dans son discours. À l'évidence, l'hapax ne nécessite pas l'amnésie du mode de vie (social/culturel) hérité. II présuppose toujours un processus antérieur de socialisation. Pour Camille cela signifie que pour réussir sa conversion, il a dû traiter avec un moi déjà formé dans un monde antérieur et déjà intériorisé (Berger et Luckmann, 1986). Et c'est sans doute parce qu'il retrouve une continuité entre passé et présent, comme le montrent les propos suivants, que l'appropriation d'un nouveau monde devient progressivement envisageable.

« À la sortie de cette première réunion, j'ai eu encore ce réflexe d'aller dans un bar que je connaissais. Sans que je demande quoi que ce soit, il (le serveur) m'a apporté un double whisky. Et du revers de la main, j'ai envoyé balader son double whisky à l'autre bout du comptoir. Et je lui ai commandé un jus d'orange. Cela a été une semaine où j'allais tous les jours en réunion. Et là, j'avais repéré un mec qui avait un nœud papillon, "bon chic bon genre", sûrement responsable d'entreprise comme moi et je lui ai demandé son numéro de téléphone.... »

On voit ici comment l'identité n'est jamais radicalement transformée. Camille s'est projeté dans un nouveau monde grâce, entre autres, à un fragment de son identité passée et présente, qu'il découvre chez cet homme «bon chic bon genre ». Un homme comme lui. Ce qui est ici à l'œuvre s'apparente de toute évidence au processus de « socialisation anticipatrice » auquel recourt R.K. Merton pour évoquer la mobilité sociale. « Du point de vue de l'individu qui adopte les valeurs d'un groupe auquel il désire appartenir, cette tendance l'aide à se hisser dans ce groupe » (Merton, 1997, p. 223). La projection dans le groupe auquel l'individu aspire apparaît comme un élément clé dans le processus qui conduit à l'arrêt définitif et durable de boire. Cette perspective est néanmoins subordonnée à un autre élément, consubstantiel au processus en question, le choc ontologique, c'est-à-dire le fait de se trouver confronté à une situation qui transforme fondamentalement l'individu : l'être qu'il croyait être n'est plus le même, l'image qu'il a de lui et des autres dans la société se déchire ; sa place, ses goûts, rien ne va plus de soi. 
Si les individus ont besoin de se retrouver dans un nouveau monde pour y rester, faut-il encore qu'ils l'aient décidé, qu'ils y soient incités, invités, autorisés, en somme que les conditions d'y participer soient réunies (Becker, 1985). Quelles sont les raisons qui poussent Camille à ce changement ? Comment expliquer ce souhait de transformation ? Toutes les personnes devenues abstinentes rencontrées au cours de nos observations chez les AA n'infirment pas l'idée selon laquelle l'arrêt soudain de boire, comme toutes les inflexions de trajectoire de vie, est souvent associé et déclenché par des événements dramatiques comme la maladie, la déception sentimentale, la mort d'un parent, etc., qui engagent un bouleversement de sociabilité, jouant un rôle dans le développement des interactions qui forment la vie sociale et l'identité. En passant au crible les entretiens, il semblerait que pour Camille l'événement déclencheur dans cette conversion corresponde à une période de longue léthargie consécutive à l'exécution d'un « sale boulot » (Hughes, 1996) :

« Fin 1976, je commence à travailler dans cette société finlandaise. Pendant cette période, je buvais toujours. Le soir, en rentrant du boulot, ma voiture ne me ramenait pas directement chez moi. Elle faisait quelques détours par des bistrots. [...] J'étais responsable de toute l'activité de cette société pour la région parisienne. Cela représentait environ 800 personnes, et le challenge qui m'avait été mis entre les mains était, pour que la société puisse continuer à faire un profit suffisant, de passer de 800 personnes à 600 personnes. Mon travail consistait à mettre 200 personnes à la porte [...]. Bon, je l'ai fait, ou je l'ai fait faire. Ce n'est pas moi tout seul qui ai fait ce boulot de boucher. Un an après mon arrivée, on a eu une réunion à Lyon, avec tous les responsables régionaux, et le PDG de la filiale française annonçait qu'enfin, en France, on avait trouvé un équilibre et que l'équilibre était dû essentiellement à ce qui avait été fait dans la région parisienne. Je me dégoûtais. Les résultats étaient satisfaisants, mais mon boulot était ignoble à faire. Après cette réunion, le week-end a passé et le lundi, je n'ai pas été foutu de repartir au boulot. Je trouvais que ce que j'avais fait était dégueulasse. Je suis resté ainsi pendant quelques mois et c'est dans cette période de léthargie où, à nouveau, j'ai beaucoup bu. C'est dans cette période-là, au bout de quelques mois, que ma femme a déposé quelques documents sur ma table de nuit, concernant les Alcooliques anonymes. Dans les documents, il y avait une notion de Dieu. Je sais bien maintenant que c'est Dieu tel que chacun le conçoit, mais moi, parfaitement athée, j'ai rejeté ça, je me suis dit : "Ma femme va me faire le coup des curés." Et puis trois quatre semaines après dans un bistrot, j'ai pété les plombs. Et là, il a fallu qu'on m'enferme, que je perde une nuit ma liberté, dans une cellule de dégrisement. Là, je prends conscience que ma vie ne vaut plus rien. J'ai repensé à ces documents des Alcooliques anonymes et je me suis dit que je n'avais rien à perdre. Allons-y ! Je ne sais pas si je suis alcoolique mais je décide d'aller voir les Alcooliques anonymes... »

À la suite de l'exécution de ce « sale boulot », Camille s'installe pendant des mois dans une position où il ne veut voir personne et continue à boire de plus belle. Ce travail aura provoqué dans son psychisme une fracture qui ramène à la surface des souvenirs qu'il aurait préféré laisser dans les nimbes de 
l'oubli. C'est au cours de cette période de réflexion (au sens étymologique de « revenir, méditer sur soi ») qu'il prend conscience que sa vie ne vaut plus rien, qu'il repense à son père et se dit : « II serait triste de me voir là où je suis. " C'est aussi au cours de cette période de doute qu'il se saisit des documents relatifs aux alcooliques : « Je me suis dit que je n'avais rien à perdre. Allons-y ! Je ne sais pas si je suis alcoolique mais je décide d'aller voir les Alcooliques anonymes. C'était le 20 mars 1978. Là, je crois que j'ai beaucoup pleuré. » La rencontre avec les AA, conjuguée à d'autres facteurs, notamment la volonté de se dégager de l'emprise de l'alcool, s'apparente ici à un choc ontologique qui conduit Camille à un ajustement biographique (Kaufmann, 2008) comme remaniement existentiel augurant une nouvelle orientation dans ses choix de vie. Dans le vocabulaire des AA, cela s'appelle le « réveil spirituel ${ }^{3}$ ». Et c'est précisément cette rencontre - vécue sous le signe de la révélation - qui sera interprétée par la suite comme un élément déterminant dans l'arrêt de boire. Or, on sait que le fait qu'une interprétation possède un degré particulièrement élevé d'évidence ne prouve rien en soi quant à sa validité empirique (Weber, 1992). Si la décision de s'en sortir est nécessaire pour arrêter de boire, elle n'en demeure pas moins insuffisante ; il faut en effet que le groupe auquel l'individu aspire offre les garanties d'un accueil pérenne, que ce soit une « structure de plausibilité efficace », une base sociale, un laboratoire de trans-formation (Berger et Luckmann, 1986). Une seconde famille en quelque sorte.

\section{UNE SECONDE FAMILLE POUR S'EN SORTIR}

Pour sortir de son errance existentielle, Camille trouve dans le monde des AA non seulement une seconde famille mais aussi une croyance collective. Croyance qui devient rapidement une « puissance supérieure » faisant office de viatique et d'horizon d'espérance. Ses propos sont, à cet égard, éloquents

« J'ai recommencé, jour après jour, comme me l'avait dit cet ami. Mais mon problème, ça n'a pas été que d'enfiler des journées sans alcool. II y avait autre chose. La vie d'un alcoolique anonyme qui veut être bien après avoir été aussi mal nécessite un nouveau mode de vie. Sans alcool, il faut que je réapprenne à marcher sans la béquille de l'alcool. J'allais dans différents groupes des AA. Je peux débarquer n'importe où, en France ou ailleurs et une réunion AA, c'est chez moi... Quand j'entre dans une réunion, quand j'en sors, je vais encore mieux. C'est vraiment un lieu où je vais recharger mes batteries. J'ai besoin de ça. C'est ma vie maintenant. C'est peut-être mon médicament. »)

Ainsi, les us et les rites spécifiques au monde des AA permettent à Camille, comme sans doute à tous les $A A$, de se reconnaître dans une communauté de sens. En créant les conditions d'un état d'attention (au sens de « tendre son esprit vers » l'autre), de disponibilités psychiques et émotionnelles, la

\footnotetext{
3 . L'idée du réveil spirituel est explicitement énoncée dans la douzième étape du code des AA : «Ayant connu un réveil spirituel comme résultat de ces étapes, nous avons alors essayé de transmettre ce message à d'autres alcooliques et de mettre en pratique ces principes dans tous les domaines de notre vie."
} 
situation de réunion permet aux uns et aux autres de se comprendre. Chacun, et avec le soutien du « modérateur ${ }^{4}$ » pour étayer l'ensemble, participe ainsi à la psychisation des tensions internes individuelles et groupales. Pour reprendre à notre compte la théorie de W.R. Bion (1961), le groupe joue ici un rôle essentiel, il participe à désintoxiquer des éléments bêta pour les modifier en éléments alpha. Ce processus de transformation des « impressions sensorielles non assimilées » (éléments bêta) en « impressions sensorielles assimilées par la psyché » (éléments alpha), rendu possible par la connivence émotionnelle, conduit progressivement les participants à faire face aux angoisses qui les tiraillent et que seule jusqu'alors la consommation d'alcool pouvait juguler. L'état d'ivresse permet en effet de faire disparaître momentanément les angoisses, les paniques, les ressentiments... Ivre, la maîtrise de soi diminue, ainsi que le besoin impérieux de se comparer aux autres. Ivre, le buveur « ressent la chaleur physiologique de l'alcool dans ses veines et, bien souvent, une sorte de chaleur psychologique à l'égard des autres. Il peut devenir larmoyant ou coléreux, mais au moins, il se sent faire à nouveau partie de la comédie humaine » (Bateson, 1977, p. 287).

En posant le principe selon lequel les interactions nécessitent un cadre, le temps des réunions instaure un langage commun. Ce langage transcende et unit les individus sans pour autant qu'il y ait interconnaissance a priori. Comme dans un rituel initiatique, à force de répétition, chacun consent à s'accepter pour ce qu'il est : un membre de la communauté des AA. Progressivement, ce sentiment d'appartenance à un groupe - en plus d'induire une narcissisation de soi - devient une référence intime et intense que chacun peut convoquer à tout moment. Pour les AA, la participation à l'élaboration d'un groupe ainsi que la contribution au maintien de sa dynamique concourent également - et sans doute davantage - à accuser individuellement réception de soi pour se prendre en charge et s'assumer en propre. Les travaux de Kaës (2000) sur la groupalité psychique sont, de ce point de vue, éclairants. Non seulement le groupe est un espace d'individuation, mais il est aussi un lieu de mise en commun des différences psychiques des éléments qui le composent. C'est grâce à cette mise en commun, appelée « appareil psychique groupal » (APG), que le groupe existe en tant qu'entité. La mise en place de l'APG est notamment rendue possible grâce à un double étayage : sur les appareils psychiques individuels des membres du groupe et sur la culture environnante et les représentations collectives du groupe. L'un des intérêts du groupe réside donc dans le fait qu'en son sein l'individu (re)joue souvent une partie de son histoire. Parce qu'il permet, grâce aux représentations et à la fantasmatisation qu'il autorise, de rejouer certaines scènes, il apparaît comme un lieu par excellence d'élaboration et de travail psychique. À l'évidence, le groupe assure ici un rôle majeur dans la consolidation des identités.

L'observation du fonctionnement des AA révèle que « pour s'associer en groupe, mais aussi pour associer des représentations et des pensées, les humains non seulement s'identifient à un objet commun et, de là, entre eux, mais encore ils scellent un accord inconscient » (Kaës, 1993, p. 266). Celles

${ }^{4}$. Chez les AA, le modérateur est celui qui est désigné par le groupe pour animer la réunion. 
et ceux qui entrent dans un groupe de $A A$ découvrent un déroulement très organisé. La réunion ressemble à une messe avec des temps de lecture s'inspirant bien souvent d'extraits des douze étapes - et de partages de représentations individuelles qui prennent la forme d'une confession collective. Tout se passe comme si entrer dans un groupe revient à participer à un collectif qui se réfère et perpétue les traditions.

Mais ces us et rites présentent un autre intérêt. Quand un AA sort des réunions, les représentations que le groupe a éveillées en lui ne s'abolissent pas une fois dehors. Elles font, extra muros, l'objet d'une déclinaison individuelle. L'histoire de Bill et Bob ${ }^{5}$, figures emblématiques des AA auxquelles il est toujours fait référence, les exploits de ceux qui racontent régulièrement leur histoire d'alcoolique et leur rencontre avec les AA comme un moment décisif restent de ce point de vue des références essentielles. Le rituel du chapeau ${ }^{6}$, la « prière de la sérénité ${ }^{7}$ », la distribution des bonbons, le temps du café, les échanges de numéros de téléphone... sont autant de pratiques qui constituent et/ou renforcent la sociabilité du groupe. Les idéaux construits collectivement en son sein « continuent à vivre dans la conscience et, par les émotions qui y sont attachées, par l'ascendant très spécial qu'ils exercent, ils se distinguent nettement des impressions vulgaires qu'entretient en lui son commerce quotidien avec les choses extérieures » (Durkheim, 1998, p. 376). À bien y regarder, nous avons tous des rituels dans notre vie. Ils permettent de la rythmer, de la structurer. Ils permettent parfois de (se) rassurer en établissant des repères, des balises, un ordre de tâches à effectuer. Un autre intérêt du rituel a trait à sa dimension contenante et rassurante. Il a en effet pour fonction de nouer des relations de qualité entre les AA et d'établir entre eux la « confiance de base »(Erikson, 1972) nécessaire à l'émergence du sentiment qu'ils peuvent compter les uns sur/pour les autres. C'est donc la solidité et la qualité du cadre contenant, assurés par le groupe, qui offrent aux AA la possibilité d'oser s'exposer, se raconter, se dire sans risque d'effraction. Les AA ne font rien de différent quand ils investissent ces rituels dans leur quotidien : par là, ils maintiennent en l'état « le système de sécurité de base » (Javeau, 1998).

\footnotetext{
5 . La naissance des AA remonte à 1935, à Akron, en Ohio, et résulte de la rencontre de Bill W, un agent de change, avec le $D^{r}$ Bob $S$, un chirurgien. Tous les deux avaient un passé d'alcooliques. Quand $D^{r}$ Bob et Bill se rencontrèrent, aussitôt, ils se mirent au travail auprès d'alcooliques de l'hôpital d'Akron ; bientôt, un premier patient trouvait l'abstinence. Le nom des Alcooliques anonymes n'avait pas encore été lancé, mais ces trois hommes formaient déjà le noyau du premier groupe des AA. À l'automne de 1935, un deuxième groupe prenait lentement forme à New York. Un troisième voyait le jour à Cleveland en 1939.

${ }^{6}$. La réunion se termine toujours par une quête nommée : " faire passer le panier » ou « faire tourner le chapeau ". Selon la septième tradition, "tous les groupes devraient subvenir entièrement à leurs besoins et refuser les contributions de l'extérieur ». Le rituel du chapeau s'apparente à un don financier des participants qui n'est pas obligatoire mais qui est rappelé à la fin de chaque réunion. Chaque participant donne en moyenne entre 1,5 et 2 euros. Dans le fonctionnement des AA, la participation financière des membres d'un groupe permet de préserver l'autonomie des AA.

7. À l'issue "du rituel du chapeau ", les participants récitent, souvent en se tenant par la main, la prière de la sérénité : "Que Dieu nous donne la sérénité d’accepter les choses que nous ne pouvons pas changer, le courage de changer celles que nous pouvons changer et la sagesse de pouvoir en saisir la différence." "
} 
Camille passe ainsi d'une absence d'amers identitaires à une vie solidement étayée par le monde des AA. De la position d'individu marginal, il devient membre à part entière de la communauté. Les errances en Alcoolie ${ }^{8}$ se transforment en un monde balisé et contenant. Pour Camille, comme pour nombre d'autres, le groupe des AA se substitue à une famille qui porte, guide et autorise à garder la tête haute, à regarder les autres dans les yeux - sans crainte (Le Breton, 2003 ; Simmel, 1981) -, et à se sentir fondamentalement l'égal de tous (Honneth, 2002). Le sentiment d'appartenance, de reconnaissance favorise le développement du sentiment de respect de soi et nourrit en retour la vie du groupe. Venir aux réunions, épouser un rôle le temps de la réunion, c'est se rattacher à l'hypothèse qu'on appartient (encore) à une communauté, autrement dit que l'on existe aux yeux des autres. On le voit, « le groupe mobilise des processus psychiques et des dimensions de la subjectivité que ne mobilisent pas, ou pas de la même manière ni avec la même intensité les dispositifs individuels » (Kaës, 2004, p. 9).

\section{LE SEUIL DU GROUPE}

L'initiation à une carrière (Becker, 1985) d'abstinence se fait par un double frottement culturel et social : en adhérant aux principes fondamentaux de la culture AA (les douze étapes, les douze traditions...) et en participant aux réunions. Ces deux types de frottement s'articulent dans le cadre d'un rite de passage renouvelé qui permet l'initiation d'un processus d'abstinence continue. Dans sa définition du rite de passage, Arnold Van Gennep (1909) présente trois temps : la séparation avec un précédent environnement ; la liminarité comme seuil entre le nouveau monde de référence et l'ancien ; l'agrégation comme le moment d'intégration au nouvel environnement social. Le rite de passage est appréhendé comme le basculement d'un environnement culturel vers un autre. Si l'anonymat symbolise le seuil, le liminaire de chaque réunion, l'alcoolique apprend à jouer sur différents registres de son identité pour passer de l'espace public à celui des Alcooliques anonymes. Dans le premier espace, son identité de AA n'est pas visible. Au sein des $A A$, son identité n'est pas révélée, à moins que la personne en juge autrement. La réunion s'apparente donc à un espace-temps séparé des autres mondes. Et c'est précisément cet entre-soi régulièrement renouvelé qui permet à Camille, comme à tous les $\mathrm{AA}$, d'entrer dans une démarche d'abstinence progressive, qui se traduira, in fine, par constituer une carrière.

Pareil à une coquille, l'espace des réunions se referme sur lui-même et inhibe temporairement la sensation désagréable d'être regardé et perçu comme un « alcoolique ». Là, il devient possible de goûter à une sociabilité sans fard et chacun, ici, traite son « prochain » avec déférence. Le grégarisme semble nécessaire pour parler la même langue et effectuer tous en même temps les rituels qui sécurisent et permettent de coexister. Pour chacun, la rencontre avec les AA a pour effet d'estomper la sensation de solitude. Le regard emphatisant de l'autre restaure la sensation d'appartenance à un groupe. De

8. Terme utilisé par certains AA pour désigner le pays dans lequel vivent les alcooliques. 
la position d'individu marginal, chacun devient au moment des réunions un membre à part entière de la « communauté des frères » (Freud, 1921) des AA. À la manière d'un sanctuaire, le temps de la réunion fait office d'un espace protégé et réservé où l'on peut être entre soi, «baisser la garde » pour se dire, dire ses souffrances et ses joies sans crainte d'être jugé. La réunion s'apparente à bien des égards à une école de moralité (Durkheim, 1902), c'est-à-dire un lieu où se fabriquent l'esprit de discipline, une âme collective, nécessaire à faire groupe. Elle est l'instance qui rappelle une regulae magistra vitae ${ }^{9}$ (comme disaient les Bénédictins) que les AA résument parfois sous l'aphorisme suivant : « Vivre un jour à la fois ; hier n'est plus demain. »

\section{UNE PUISSANCE SUPÉRIEURE POUR TENIR LE COUP}

Le groupe joue donc un rôle en matière d'éducation à la désalcoolisation. La participation aux réunions est l'occasion pour chacun de redécouvrir les contours de soi, à commencer par la parole. Les expériences partagées initient des solidarités. Celles-ci nourrissent le processus de conversion. Là, l'individu est tenu - de l'extérieur - par un ensemble de forces, parfois indépendamment de sa volonté (Martuccelli, 2002). Camille en atteste : « Avant de rencontrer les AA, je vivais échec sur échec. J'étais insatisfait. Je me sentais seul et je pensais être seul à vivre ce type de vie... » Parce qu'il rassure, parce que la parole y est écoutée, libre, le groupe apparaît donc au travers d'une représentation positive des AA - comme un lieu d'étayage des identités. En ce lieu, l'autre devient une version possible de soi. Les expériences partagées de parcours chaotiques plus ou moins similaires font incontestablement thérapie en ce sens qu'elles sont l'occasion pour chacun non seulement de penser l'autre comme un soi-même, mais aussi et surtout de se penser soi-même comme un autre (Ricœur, 1995). Répétées dans le temps, ces situations placent les individus en position de faire l'expérience de l'échoïsation corporelle et cognitive (Bergé et Cosnier, 2003). Le corps et la parole servent alors d'instrument d'analyse des affects d'autrui. Le passage du ressenti à la parole contribue à revitaliser l'autre, à lui rendre sa place de sujet digne de respect. C'est probablement parce que chacun parle de ce qui lui tient à cœur et que cela rencontre un écho chez tous les autres que le groupe - lieu de mise en commun des images intérieures - devient un espace d'étayage des identités groupales et individuelles (Anzieu, 1999). Le groupe des pairs, lieu de socialisation, espace irrésistible, occupe ainsi une place primordiale dans le processus de conversion parce qu'il fonctionne comme un espace d'influence réciproque. Les objets, au même titre que la parole, échangés dans une dynamique de réciprocité nourrissent en retour la reconnaissance mutuelle (Sahlins, 1976). Pour Camille, comme beaucoup de AA, les réunions remplissent une fonction essentielle : celle d'un sentiment d'appartenance collective. Les règles, les rituels et toutes les croyances donnent au temps de réunions une dimension solennelle comparable à une cérémonie religieuse favorisant l'avènement d'une communion des consciences qui constitue, in fine, " la meilleure manière de s'attester soi-

9 . Maître-programme de vie. 
même et d'attester à autrui qu'on fait partie d'un même groupe » (Durkheim, 1998, p. 333). Les phénomènes générés par le groupe rappellent qu'il n'est ni la simple sommation des individualités ni le résultat de volitions communes. II est, selon Eugène Enriquez, bien plus que cela. II ne s'agit pas, en effet, « de vouloir ensemble, il s'agit de sentir ensemble, d'éprouver la même nécessité de transformer un rêve ou un phantasme en réalité quotidienne... Tout groupe fonctionne à l'idéalisation, à l'illusion, à la croyance » (Enriquez, 1992, p. 101).

À propos de croyance, dans la présentation des douze étapes, il est fait mention d'une puissance supérieure, qui se révèle être « Dieu tel que nous le concevions ». Le chiffre douze, des douze étapes et des douze traditions, n'est pas sans rappeler les douze apôtres du Christ. Ces différents indices, ainsi que les rituels des AA (lecture du « Préambule des AA »; moment de silence ; lecture d'un extrait du Gros Livre ; prière de la sérénité) peuvent laisser croire que les AA seraient une organisation religieuse. Mais la dixième tradition précise que les AA n'expriment aucune opinion sur des sujets étrangers et aucune croyance religieuse n'est nécessaire de la part des personnes qui entrent dans un groupe : "Parmi les membres, on trouve des catholiques, des protestants, des Juifs, des représentants de toutes les autres religions, ainsi que des agnostiques et des athées » (Fascicule des « 44 questions » des AA). En revanche, comme le révèle la lecture des étapes, le programme de rétablissement est sans ambiguïté basé sur l'acceptation de certaines valeurs spirituelles, mais précise - comme pour appeler à l'œcuménisme - que " chaque membre est libre d'interpréter ces valeurs comme il l'entend, ou de les ignorer ».

Ces fondements religieux du programme des AA se trouvent en germe dans les confessions des deux fondateurs. Bill était protestant, Bob catholique. À la réflexion, «l'ensemble du système des AA est une religion durkheimienne, au sens où la relation entre l'homme et sa communauté est parallèle à la relation entre l'homme et Dieu » (Bateson, 1977, p. 292). Ce « Dieu tel que nous le concevons », Camille en fait un syncrétisme qui lui permet de s'inventer « sa » propre croyance. Sa puissance supérieure n'est pas le Dieu de Bill ou de Bob. Athée, ou plutôt n'ayant pas la foi catholique en l'existence de Dieu, il dit avoir cependant accepté l'idée que pour arrêter de boire, il fallait « croire que cela fût possible ». À sa façon, Camille dévoile sa foi en l'abstinence, dans une acception profane.

\section{CONCLUSION}

Les analyses du discours de notre interlocuteur révèlent que le monde des AA est une communauté de sens. Vivre à l'intérieur d'un monde, quel qu'il soit, demande une affiliation à une entité. Le processus - dont l'hapax n'est qu'un moment dans un ensemble complexe - qui conduit Camille à devenir abstinent aura été rendu possible par la communauté des AA qui le respecte et, point essentiel, lui rend sa dignité. La découverte du monde des AA aura été pour lui, et sans doute pour nombre de $A A$, une opportunité pour revoir et abandonner certains attributs constitutifs d'anciennes identifications et pour envisager d'autres horizons. Ce travail de subjectivation qui s'accompagne 
d'un profond remaniement interne est comparable à une renaissance, au sens étymologique de « régénération spirituelle ».

L'exemple de Camille n'est bien entendu pas représentatif de l'ensemble des personnes qui participent aux réunions ou qui se revendiquent AA. Mais audelà des variations, parfois importantes, entre les trajectoires d'abstinence, ce cas demeure cependant idéal-typique d'une partie des AA rencontrés. Ce travail sur la vie de Camille montre que le groupe des AA est un outil dont la fonction sociale complète la palette des modalités permettant à un individu d'envisager d'arrêter de boire. Les AA ne sont pas un remède universel. Du reste, la prise en charge médicale et/ou psychologique ne l'est pas non plus. Et le fait d'assister régulièrement à un groupe des AA n'est pas toujours la solution unique à un problème individuel aux multiples facettes. Certaines personnes suivent le programme des AA depuis une dizaine d'années, entre des séjours réguliers dans des centres de cure. D'autres viennent en réunion en étant alcoolisées. Si l'abstinence est une solution pour Camille c'est moins parce qu'il se reconnaît dans l'idéologie des Alcooliques anonymes que parce qu'il s'est redécouvert en tant qu'individu en mesure de se fixer des objectifs, de retrouver une part essentielle de vie dans la reconnaissance sociale.

Ce que proposent les Alcooliques anonymes est un programme, libre, dont l'usage est gratuit, sans contrainte, dont chacun peut utiliser tout ou partie. Pour Camille, comme pour Jérôme, Jean-Luc, Pascaline et bien d'autres, c'est un modèle structurant qui permet à chacun de se construire son abstinence en sens et en actes sans jamais, point essentiel, sortir l'alcool de sa vie. Ce qui nous amène à laisser Michel Legrand conclure : « Le membre d'un groupe d'anciens buveurs, d'autant plus qu'il est militant, n'a pas sorti l'alcool de sa vie, au contraire il ne cesse d'y penser et lui consacre tous ses instants libres. Il s'agit moins là d'une guérison - mais, encore une fois, les AA n'ont pas cette prétention - que de conversion. Mais quelle serait donc la véritable guérison ? » (Legrand, 1997, p. 91).

\section{BIBLIOGRAPHIE}

ANZIEU, D. 1999. Le groupe et l'inconscient, Paris, Dunod.

BARBIER, R. 1997. L'approche transversale, Paris, Anthropos.

BATESON, G. 1977. Vers une écologie de l'esprit, Paris, Le Seuil.

BECKER, H.S. 1985. Outsider : étude sociologique de la déviance, Paris, Métailié.

BERGÉ, C. ; COSNIER, J. 2003. « Empathie et autisme : de l'analyseur corporel à la clinique de l'empathie », dans CALAP (Cahiers d'acquisition et de pathologie du langage), $\mathrm{n}^{\circ} 23$, université René-Descartes.

BERgER, P. ; LUCKMANN, T. 1986. La construction sociale de la réalité, Paris, Méridiens Klincksieck et Cie.

BeRTAUX, D. 1997. Les récits de vie. Perspective ethnosociologique, Paris, Nathan.

BION, W R. 1961. Recherches sur les petits groupes, Paris, Puf, 1965.

DURKHEIM, É. 1902-1903. L'éducation morale, Paris, Fabert, 2005.

DURKHEIM, É. 1912. Les formes élémentaires de la vie religieuse, Paris, Puf, 1998.

DURKHEIM, É. 1977. Les règles de la méthode sociologique, Paris, Puf, 1995.

ELIAS, N. 1991. La société des individus, Paris, Fayard.

ENRIQUEZ, E. 1992. L'organisation en analyse, Paris, Puf. 
ERIKSON, E. 1972. Adolescence et crise. La quête de l'identité, Paris, Flammarion.

FoucAult, M. 1973. Moi, Pierre Rivière, ayant égorgé ma mère, ma sœur et mon frère, Paris, Gallimard.

FREUD, S. 1921. "Psychologie des foules et analyse du moi », dans Essais de psychanalyse, Paris, Payot, 1989.

GARFINKEL, H. 1967. Recherches en ethnométhodologie, Paris, Puf, 2007.

HOFFMAN, M. 2008. Empathie et développement moral, Presses universitaires de Grenoble.

HoggaRT, R. 1991. Autobiographie d'un intellectuel issu des classes populaires anglaises, Paris, Le Seuil.

HONNETH, A. 2002. La lutte pour la reconnaissance, Paris, Cerf.

HUGHES, C.H. 1996. Le regard sociologique, Paris, EHESS.

JANKÉLÉVITCH, V. 1957. Le je-ne-sais-quoi et le presque-rien, Paris, Puf.

JAVEAU, C. 1998. Prendre le futile au sérieux, Paris, Éditions du Cerf.

KAËS, R. 2000. L'appareil psychique groupal, Paris, Dunod.

KAËS, R. 2000. Théories psychanalytiques du groupe, Paris, Puf.

KAËS, R. 1993. Le groupe et le sujet du groupe, Paris, Dunod.

KAUfMANN, J.-C. 2008. Quand Je est un autre, Paris, Nathan.

KESSEL, J. 1960. Avec les alcooliques anonymes, Paris, Gallimard.

LE BRETON, D. 2003. Des visages, Paris, Métailié.

LE BRETON, D. 2010. Expérience de la douleur, Paris, Métailié.

LEGRAND, M. 1993. L'approche biographique, Paris, Desclée de Brouwer.

LEGRAND, M. 1997. Le sujet alcoolique, Paris, Desclée de Brouwer.

MARTUCCELLI, D. 2002. Grammaires de l'individu, Paris, Gallimard.

MATZA, D. 1964. Delinquency and Drift, New York, Wiley.

MERTON, R.K. 1997. Éléments de théorie et de méthode sociologique, Paris, Armand Collin.

MILLS, C.W. 1983. L'imagination sociologique, Paris, Maspero.

NiETZSCHE, N. 1908. Ecce homo, Paris, Mille et une nuits, 1999.

PENEFF, J. 1990. La méthode biographique, Paris, Armand Colin.

RICEUR, P. 1995. Le juste, Paris, Esprit, 1995.

SAHLINS, M. 1976. Âge de pierre, âge d'abondance, Paris, Gallimard.

SCHÜTZ, A. 1987. Le chercheur et le quotidien, Paris, Méridiens-Klinckieck.

SIMMEL, G. 1981. Sociologie et épistémologie, Paris, Puf.

THOMAS, W.I. ; ZNANIECKI, F. 1919. Le paysan polonais en Europe et en Amérique : récit de vie d'un migrant, Paris, Nathan, 1998.

VAN GENNEP, A. 1909. Les rites de passage : étude systématique, Paris, Éditions Nourry, 1981.

WEBER, M. 1904. Essais sur la théorie de la science, Paris, Agora Pocket, 1992.

WEBER, M. 1919. Le savant et le politique, Paris, UGE, 1963.

WHYTE, W.F. 1943. Street Corner Society. La structure sociale d'un quartier italo-américain, Paris, La Découverte, 1995.

ZANNA, O. 2010. « Un sociologue en prison », Nouvelle revue de psychosociologie, $\mathrm{n}^{\circ}$ 9, p. $149-162$.

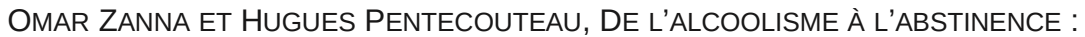
LE CHOC BIOGRAPHIQUE COMME RUPTURE SYMBOLIQUE

RÉSUMÉ

Nous proposons de rendre compte d'une recherche menée pendant deux ans dans le milieu des Alcooliques anonymes. Parmi l'ensemble des AA rencontrés, la plupart font référence à plusieurs facteurs étroitement mêlés pour expliquer leur changement 
radical d'habitude et de vision du monde. Cela dit, l'analyse du matériel recueilli a rapidement révélé que ce changement radical était en réalité à considérer comme le fruit d'un processus complexe qui ne peut se réduire à un événement spontané et individuel se produisant ex nihilo, comme beaucoup d'AA semblent le penser. Pour ces personnes qui ont - après la rencontre avec les AA - rapidement arrêté de boire, l'abstinence s'apparente à une forme d'« insight » - c'est-à-dire la découverte soudaine de la solution à un problème qui fait naître brusquement un cheminement radicalement différent. Or, l'arrêt de boire est un processus qui nécessite tout un cheminement en amont et en aval de la rencontre avec les AA. Pour rendre compte de ce processus, nous avons fait le choix de nous intéresser à un " cas », parmi d'autres: Camille. Camille est un AA âgé de 80 ans, abstinent depuis plus de trente ans. En optant pour le récit biographique, il s'agit de partir d'une histoire personnelle afin d'y repérer un cheminement classique. Se concentrer sur un cas extrême de changement d'être-au-monde permet de mettre en saillance les éléments du processus du devenir que l'on retrouve dans d'autres cas d'alcoolo-dépendance. L'analyse en profondeur d'un cheminement ou d'un phénomène livre exactement les mêmes aperçus que ceux obtenus par l'analyse de faits relativement superficiels illustrant l'étendue d'un grand nombre de cheminements ou de phénomènes.

\section{MOTS-CLÉS}

Alcoolisme, Alcooliques anonymes, abstinence, groupes.

OMAR ZanNa, Hugues PENTECOUTEAu, From ALCOHOlism to SOBRIETy : THE BIOGRAPHIC SHOCK AS A SYMBOLIC BREAK

\section{ABSTRACT}

The purpose of this paper is to give account of a research based on a two years fieldwork in the Alcoholics Anonymous circle. Amongst all the AA met, most explain their radical change of habits and way of life by several tightly linked factors. Yet, analysis of the collected material has quickly revealed that this radical change had to be understood as the result of a complex process which can not be reduced to a spontaneous and individual « coming out », as many AA seem to believe. For these persons who gave up drinking, soon after having met with the AA, abstinence is similar to a kind of « insight »- ie the sudden discovery of a solution to a problem, that leads to a radically new way of life. Therefore, giving up drinking is a long process, which requires a progression before and after meeting with the AA. To give account of this process, we chose to focus on a "case », among others : Camille. Camille is an 80 year old AA, abstemious practically from one day to an other, for more than 30 years and after about thirty years of alcoholism. Choosing the biographic account consists in discovering the signs of a classical path in an individual history. Focusing on an extreme case of changing their being-in-the-world allows the saliency of element of the process of belonging found in less drastic cases. The deep analysis of one case or one phenomenon gives equivalent results to those obtained with a lighter analysis of several cases or phenomena.

\section{KEYWORDS}

Alcoholism, alcoholics Anonymous, sobriety, groups. 